\title{
Increased Risk of Coronary Heart Disease in Patients with Anxiety Disorders: A Review of Underlying Biomarkers
}

\author{
Samuel Cyr ${ }^{1,2}$, Ariane-Sophie Painchaud-Bouchard ${ }^{1,3}$, Marie-Joëlle Marcil ${ }^{1}$, David Rhainds ${ }^{1}$ and Judith \\ Brouillette ${ }^{1,4 *}$ \\ ${ }^{1}$ Research Center, Montreal Heart Institute and Université de Montréal, Canada \\ ${ }^{2}$ Faculty of Pharmacy, Université de Montréal, Canada \\ ${ }^{3}$ Faculty of Medicine, Université de Montréal, Canada
}

${ }^{4}$ Department of Psychiatry and Addiction, Université de Montréal, Canada

*Corresponding author: Judith Brouillette, Montreal Heart Institute, 5000 Belanger Street, Montreal, Quebec, H1T 1C8, Canada

\section{ARTICLE INFO

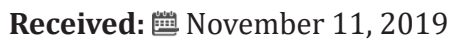 \\ Published: ${ }^{\mathrm{w}}$ November 19, 2019}

Citation: Samuel Cyr, Ariane-Sophie Painchaud-Bouchard, Marie-Joëlle Marcil, David Rhainds, Judith Brouillette. Increased Risk of Coronary Heart Disease in Patients with Anxiety Disorders: A Review of Underlying Biomarkers. Biomed J Sci \& Tech Res 23(1)-2019. BJSTR. MS.ID.003844.

\section{ABSTRACT}

Anxiety is the most common mental health disorder in the United States, affecting nearly a third of the population. As for depression, it is associated with increased risk of incident coronary heart disease (CHD) and poor cardiac prognosis. The pathophysiological mechanism underlying this "deleterious" association is not well defined. While several hypotheses have been proposed, few seem proven in original studies. A narrative literature review was thus performed to identify all original studies that looked at any biomarkers that can be implicated in the relation between anxiety and CHD. Surprisingly, only four cohorts or observational studies on anxiety and CHD reported biophysiopathological variables. Of the overall populations studied, only $15 \%$ were women. In term of biomarkers, plasma lipid levels, C-reactive protein, cortisol, norepinephrine, body mass index, blood pressure and heart rate variability were mostly not significantly different between anxious patients and controls. Only two variables, myocardial perfusion and coronary artery calcium, were found different between the two groups. In summary, underlying biomarkers explaining the increased risk of CHD in anxious patients are still poorly understood. Although based on very limited data, myocardial perfusion and coronary artery calcium seem to be plausible biomarkers. Clearly, more studies are needed to better understand this problematic, especially in women. This step is essential so that personalized care for patients with both anxiety and CHD can be implemented.

\footnotetext{
Abbreviations: BMI: Body Mass Index; CHD: Coronary Heart Disease; CRP: C-reactive Protein; DBP: Diastolic blood pressure; DSM: Diagnostic and Statistical Manual of Mental Disorders; GAD: Generalized Anxiety Disorder; HDL: High-Density Lipoprotein; HRV: Heart Rate Variability; LDL: Low-Density Lipoprotein; MACE: Major adverse cardiac events; NE: Norepinephrine; PTSD: Posttraumatic Stress Disorder; SBP: Systolic Blood Pressure; TG: Triglyceride
} 


\section{Introduction}

Anxiety disorders are the most common mental health disorders in the United States (28.8\%) [1] and are even more prevalent in patients with cardiovascular disease (CVD)(37\%) [2]. The relationship between anxiety and cardiovascular disease is complex and bidirectional. On one side, having a cardiovascular disease can lead to anxiety. On the other side, having anxiety or Posttraumatic Stress Disorder (PTSD) is associated with both incident Coronary Heart Disease (CHD) and poor cardiac prognostic $[3,4]$. Considering CHD is the primary cause of death in the USA [5] and neuropsychiatric conditions are the leading cause of invalidity [6], this "deleterious" association is a major social concern. Surprisingly, there are only a few studies regarding the link between confirmed anxiety disorders and CHD development.

Most studies seem to target depression, hostility, or stress. Moreover, although several hypotheses linking anxiety to cardiovascular diseases have been proposed [7-10], the physiopathological mechanisms responsible for linking these two pathologies remain to be specified. This step is crucial so that research can evolve to explore novel treatment options that may be more specific/personalized for patients suffering from both anxiety and CHD. Fortunately, in the field of depression, two recent studies point out that untreated depression, rather than depression itself, is associated with poorer cardiac outcomes $[11,12]$. Underlying mechanisms explaining a poor CHD prognosis have also been mostly studied in depression or self-reported stress. To illustrate the relative contribution of scientific research in each field, an advanced search in MEDLINE with "depression, CHD and biomarker" yielded 3500 articles. The number rose to 7289 when the term "depression" was replaced by "stress."

However, when using terms "anxiety disorders" and/or "PTSD," only 205 articles were found (for ease of reading, only anxiety disorders will be mentioned from now on and will include PTSD). This suggests that clinical populations of patients with both anxiety disorder and CHD are understudied. It was decided to focus on confirmed anxiety disorders since some studies suggested that confirmed, and not self-reported elevated anxiety symptoms, were associated with poor cardiac outcomes [4]. More importantly, confirmed anxiety disorder increases homogeneity across studies since elevated anxiety on self-reported questionnaires can represent personality suffering, for example. Inflammatory markers $[13,14]$, thyroid hormones $[15,16]$, hypothalamic-pituitaryadrenal (HPA) axis [17], brain-derived neurotrophic factor (BDNF) $[18,19]$ and autonomic nervous system hormones [20] have all been documented to impact CHD prognosis as well as being altered by the presence of anxiety disorders, although in separate studies.

Furthermore, Heart Rate Variability (HRV), the physiological variation of heartbeats intervals, coronary artery calcium score and coronary flow reserve have been proposed as possible underlying biomarkers responsible for the association between anxiety and cardiovascular diseases [21-23]. A narrative review of the literature was conducted to gather all available information to clarify current understanding of this important "deleterious" association.

\section{Methods}

Although this a narrative literature review (vs. systematic), the PRISMA search strategy was used as a model to conduct this review [24].

\section{Eligibility Criteria}

The study population had to present an anxiety disorder before the cardiac endpoint was measured. The method used to assess the presence of anxiety disorder (questionnaires or interview with a psychiatrist) had to be clearly mentioned. Either infarction, coronary heart disease, coronary revascularization, Major Adverse Cardiac Events (MACE) or mortality had to be part of the outcomes studied. A biochemical marker (e.g., levels of catecholamine, inflammatory proteins) or pathophysiological functional or approximate marker (e.g., HRV, coronary artery calcium, coronary flow reserve) had to be reported in anxiety and control groups. Finally, studies or publications in the form of literature reviews or systematic reviews were excluded.

\section{Search Strategy}

Publications were identified by a search of Medline, PsycINFO and CINAHL. Exact search strategies may be found in the supplementary material. Results were restricted to publications in English or French. The first search was performed June 21, 2019, and the last search was conducted on June 27, 2019. Reference lists of relevant publications were examined by manual searches.

\section{Study Selection}

A flow diagram of the study selection process is presented in Figure 1. Research through MEDLINE, PsycINFO and CINAHL databases yielded a total of 635 articles. Among selected articles, reviews of literature or meta-analysis were examined for cross-references. After duplicates removal, titles and abstracts were screened for eligibility criteria by authors AS. PB., S.C. and MJ. M. Publications with unclear inclusion status were assessed by a third independent author (J.B.). Full text was examined for the remaining publications.

\section{Data Extraction Procedure}

We developed a data extraction template that was used by two authors (AS.PB. and S.C.) to extract the following data: type of anxiety disorders studied, method used for assessing anxiety disorders/ PTSD, number of participants, demographic characteristics (male/ female distribution, mean age), biomarkers and type of outcomes (CHD incidence, MACE or death). 


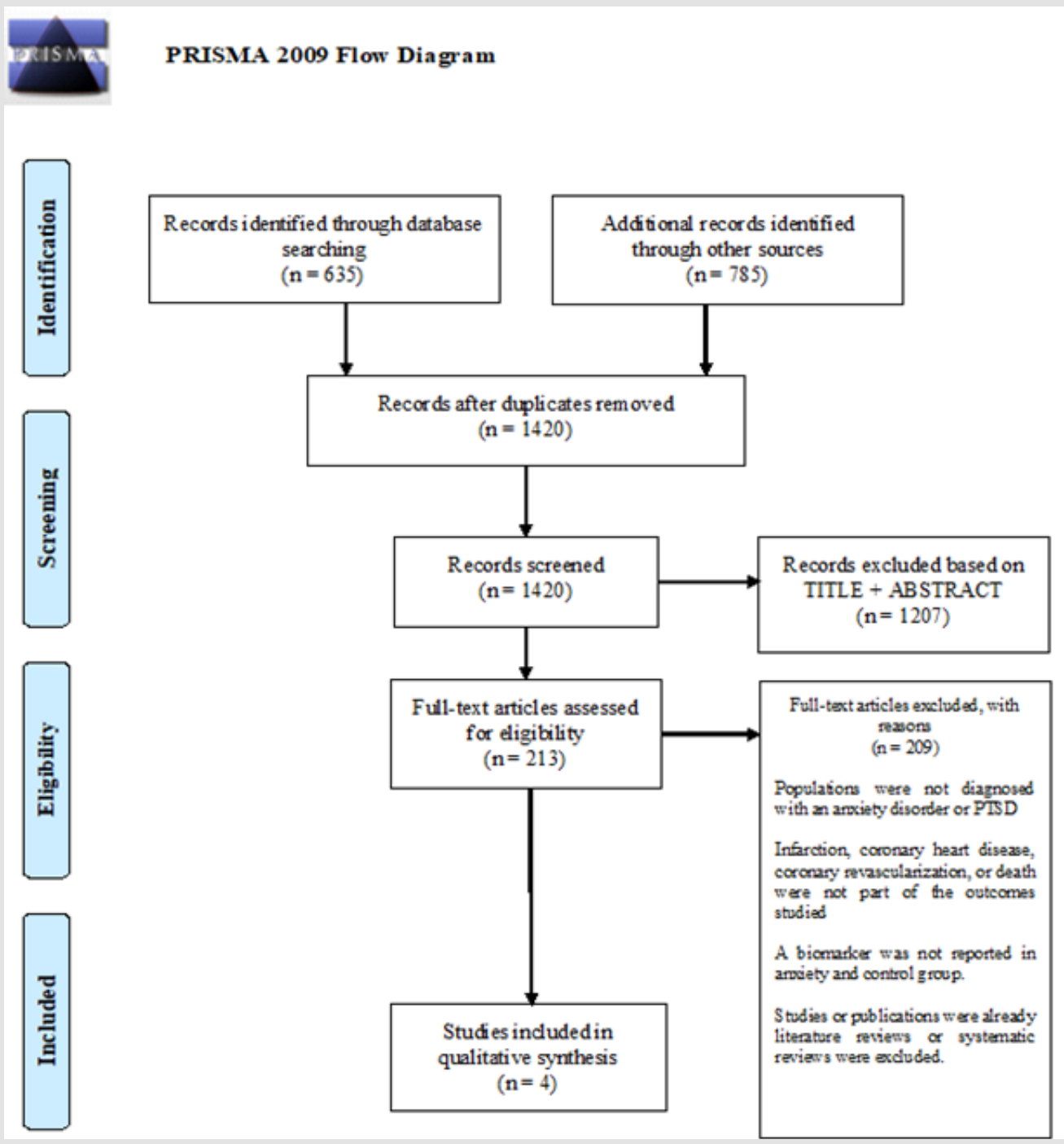

Figure 1: PRISMA flow diagram of literature review.

\section{Quality Assessment}

Two authors (AS. PB. and MJ. M.) independently assessed the risk of bias in each study with the Newcastle-Ottawa Quality Assessment Scale [25]. Each category ranged from zero (definite or unclear risk of bias) to one star (no risk of bias). Total score was then calculated; an article was deemed good quality if it had three or four stars in the selection domain, one or two stars in the comparability domain and two or three stars in the outcome/ exposure domain. We only included studies with a good quality.

\section{Results}

\section{Characteristics of Included Studies}

A total of 635 articles were found through database searches after duplicates removal. The cross-referencing allowed us to increase the number of potentially interesting articles to 1420 (785 additional articles). Figure 1 depicts the PRISMA flow diagram illustrating the study selection process [24]. 1207 articles were first excluded based on title and abstracts and 209 items were further excluded after full-text examination. Four items were thus included in our literature review. The four selected studies were either cohort or descriptive studies. Four other articles were found to be interesting in terms of biomarkers studied but did not meet the eligibility criteria; either there was no cardiac endpoint measured or it wasn't a confirmed anxiety disorder but rather anxiety symptoms. Those articles will be discussed further in the discussion section but were not included in this review.

\section{Increased Risk of Coronary Heart Disease in Anxiety Disorders Patients}

Overall, there was a significant augmentation of CHD or poor cardiac prognosis in anxiety disorder patients reported in all four articles. Frasure Smith et al. [4] showed that patients with Generalized Anxiety Disorder (GAD) and stable CAD were 2.4 times more likely to experience MACEs two years after acute coronary syndrome [Unadjusted odds ratio; 95 \% CI : 1.2 ; 5.0]. This association remained significant after adjusting for a number of covariables including age, sex, current daily smoking, and severity of CHD at 
baseline. Martens et al. [21] found an annual rate of cardiovascular events in patients with GAD of $9.6 \%$ compared to $6.6 \%$ in the patients without GAD ( $p=0.03)$. Finally, Vaccarino et al. [23] reported that the incidence of CHD was twice as high in patients with PTSD as in those without PTSD $(22.6 \%$ vs. $8.9 \%$, p < 0.001$)$.

\section{Biomarkers Studied in CHD-Anxiety Population}

Table 1 summarizes the current state of knowledge on biomarkers possibly linked to poor CHD outcome in patients suffering from anxiety disorder. Among the selected articles, 3/4 reported high-density lipoprotein (HDL) [4,21,22], 2/4 low-density lipoprotein (LDL) [21,22], 1/4 total cholesterol [22] and 2/4 triglycerides

Table 1: Summary of included studies in relation with the coronary heart disease outcome and biomarkers.

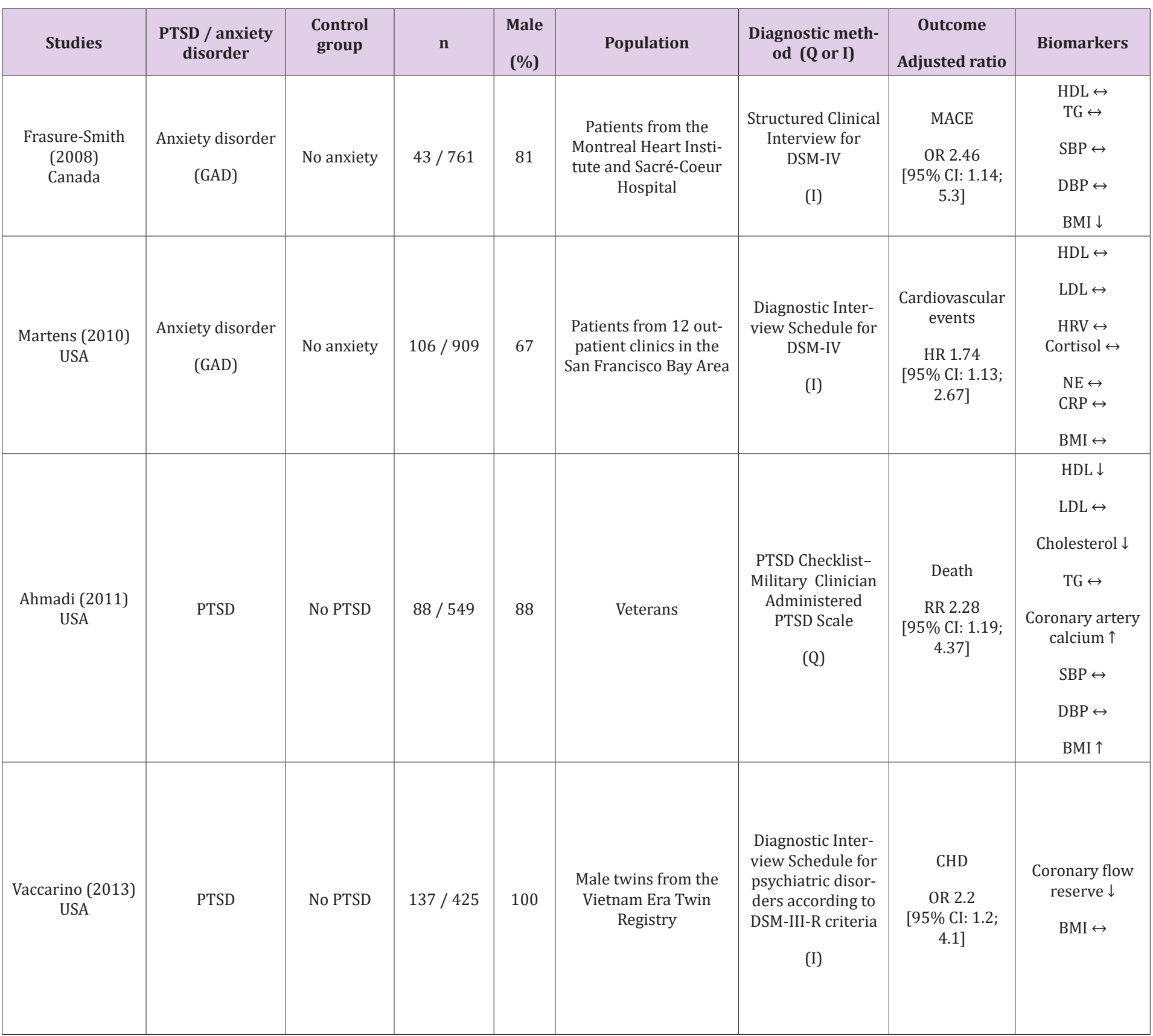

Note: Study and population characteristics (with $\mathrm{n}=$ number of patients in the population) as well as diagnostic methods are presented in this summary table. Difference between anxiety disorder/PTSD vs. control group on biomarkers $(\uparrow, \downarrow$, $\leftrightarrow)$ is presented as well as the CHD outcome.
$[4,22]$. The inflammation marker C-Reactive Protein (CRP) was found in only $1 / 4$ reference [21]; body mass index (BMI) was reported in 4/4 studies [4,21-23] while systolic and diastolic blood pressures in 2/4 studies [4,22]. 24-hour urine output of cortisol and Norepinephrine (NE) as well as HRV were reported once, in the same study [21]. One article measured coronary artery calcium in PTSD versus control patients [22] and another measured the coronary flow reserve [23]. Only $2 / 4$ papers [22,23] had a primary focus on the biomarker (coronary artery calcium or coronary flow acteristics. reserve). All other biomarkers were documented as baseline char- 


\section{Blood Lipids}

HDL, often called the "good" cholesterol [26], was not different in $2 / 3$ articles reporting HDL [4,21]. The other study showed significantly decreased HDL in patients with PTSD versus nonPTSD (38 vs. $44 \mathrm{mg} / \mathrm{dL}, \mathrm{p}=0.005$ ) [22]. LDL, the "bad" cholesterol [27], was not different between anxiety patients and controls in all 2/2 studies that measured it $[21,22]$. Total cholesterol, another risk factor for CHD [28], was mentioned in one article [22] in which levels were significantly decreased in patients with PTSD compared to controls (163 vs. $174 \mathrm{mg} / \mathrm{dL}, \mathrm{p}=0.04$ ). High level of Triglycerides (TG) is a known risk factor for the development of cardiovascular diseases [27]. The levels of TG were equivalent in patients with anxiety disorder compared with those without anxiety in all $2 / 2$ articles that reported TG levels [21,22].

\section{Cortisol, Norepinephrine and Heart Rate Variability}

Martens et al [21] showed no difference between GAD patients and control group in terms of cortisol, NE levels or HRV.

\section{Inflammation}

CRP, a systemic marker of inflammation [29] and a good predictor of cardiovascular events [30] showed no significant difference in the Martens et al study [21] in a group of patients with GAD versus controls.

\section{Coronary Artery Calcium}

Coronary artery calcium score is associated with long-term occurrence of MACE [31] and is a marker of atherosclerosis. Ahmadi et al. [22] screened 367 veterans without known CAD and showed that coronary artery calcium was significantly more prevalent in the PTSD cohort than in the non-PTSD cohort (76\% vs. 59\%, p =0.001).

\section{Coronary Flow Reserve}

One study documented coronary flow reserve, an indicator of myocardial perfusion and a known risk factor of myocardial infarction [23]. Coronary flow reserve was significantly lower in twins with PTSD compared to their unaffected brother. In terms of proportion, abnormal coronary flow reserve was more frequently reported in twins with PTSD (43.5\%) than in those without $(24.7 \%$, $\mathrm{p}<0.001$ ).

\section{Body Mass Index}

High BMI has been associated in many studies with poor longterm cardiovascular outcomes [32,33]. BMI was not statistically different in $2 / 4$ studies [21,23] was lower in $1 / 4$ study [4] and higher in another [22]. Indeed, Ahmadi et al showed that patients with PTSD had a mean BMI $(\mathrm{kg} / \mathrm{m} 2)$ of 31.0 while patients without PTSD had a mean BMI of $29.3(\mathrm{p}=0.02)$.

\section{Blood Pressure}

Hypertension is a known risk factor for cardiovascular disease [34-36]. The two papers [4,22] which reported blood pressure showed no significant difference between anxious and non-anxious patients.

\section{Discussion}

\section{Biomarkers: What Was Learned?}

This literature review was conducted in order to gain better understanding of the pathophysiological mechanisms underlying anxiety disorders/CHD interaction. Our first observation is that this question is very little studied. To our surprise, only two studies selected biomarkers of CHD in anxious patients as a primary outcome [22,23]. The other papers documented biomarkers as baseline characteristics. Our second observation is that BrainDerived Neurotrophic Factor (BDNF) and thyroid hormones have not been measured in cohort or descriptive studies of CHD and anxiety disorder patients. The most common biomarkers reported were lipids (HDL, LDL, TG, and cholesterol, and TG). Interestingly, the lipid levels of anxious patients were generally not different from non-anxious patients. Only one study had documented a change in HDL levels as well as total cholesterol [22].

Whereas greater autonomic dysfunction (reflected by reduced HRV) [37], increased catecholamines [38,39] and cortisol levels [40] have already been associated with CHD in depressed patients, this was not the case in the one study in GAD patients (no difference for all these measures) [21]. BMI and blood pressure were also usually equivalent between groups in different studies and based on the evidence currently available, cannot explain the development of CHD in anxious patients. Furthermore, authors noticed that very few women were studied through the four included references, with an average female percentage as low as $15 \%$. This difference is even more unaccountable given that women are up to twice as likely to have an anxiety disorder as men [41]. As heart disease is the top killer in women [42] and that there is a known gender difference in the clinical presentation [43], it will be important in the future to include more women in the studies in order to investigate a more representative population.

\section{Hints from other studies}

Some excluded studies (absence of reported coronary heart disease, MACE or death overtime) compared baseline characteristics of anxious versus non-anxious participants and calculated theoretical 10-year risk of CHD or odds ratio of different outcomes. Dzubur Kulenovic et al. [44] reported levels of different lipids. LDL, TG and total cholesterol were significantly higher, and HDL lower, in PTSD patients. Their theoretical ten-year risk was significantly higher than the group without PTSD (19.4 vs 9.7, $\mathrm{p}<0.001$ ). This is expected because the lipid levels suggest increased risk (low HDL, high LDL and TG) Solter et al. [45] found similar results with regards to lipids measurement between PTSD and major depressive disorder patients as compared with PTSD patients having degraded lipid profile. Turner et al. [46] studied myocardial ischemia as outcome between PTSD and non-PTSD patients. Patients with a diagnosis of PTSD were 2.4 times more likely to have myocardial ischemia compared to patients without PTSD [Adjusted odds ratio; 95\% CI: 1.3; 4.2]. At baseline, plasma lipid levels were equivalent, except for higher triglycerides in the 
PTSD group. Also, CRP levels were increased in PTSD patients (2.7 vs. $2.4 \mathrm{mg} / \mathrm{L}, \mathrm{p}=0.02$ ).

Shen et al. [47] showed that each standard deviation (SD) increase in anxiety levels predicted myocardial infarction incidence with a multivariate relative risk of 1.4 [95 \% CI: 1.2; 1.8]. In their paper, there was no significant difference between anxious and non-anxious patients for fasting glucose. It is known that impaired fasting glucose is a predictor of coronary heart disease [48]. It was also not significantly correlated with overall anxiety. A slightly lower pressure in anxious patients compared to non-anxious patients was also reported. They demonstrated a significant $(\mathrm{p}<0.05)$ negative correlation with overall anxiety for both systolic and diastolic blood pressure ( -0.08 and -0.09 , respectively) with a mean blood pressure of 128.7 over $78.7 \mathrm{~mm} \mathrm{Hg}$.

Unfavorable lipid profile and CRP level reported in these studies are conflicting with results found in the narrative literature review. To explain this difference, two hypotheses have been put forward. In the first place, it was thought that patients had potentially less optimal lifestyle habits (smoker, alcohol consumption, sedentary lifestyle, etc.) in studies with unfavorable profile versus favorable profile. This hypothesis was proved false after verification as populations were similar in terms of lifestyle. Also, as the different biomarkers were calculated either as basic characteristics or as main outcome, insufficient statistical power could have contributed to the difference. Once again, this hypothesis was infirmed as number of patients was similar between studies.

\section{Limitations}

The exact search strategy used by the authors was carried out using biomarkers known to have been studied in the field of anxiety and heart disease. Therefore, articles that dealt with biomarkers that the authors did not know about may have been missed out.

\section{Conclusion}

There is no doubt that CHD and MACE are more important in patients with anxiety, an association repeatedly proven through different studies. The literature is however very sparse in relation to possible biomarkers that could explain the occurrence of longterm heart disease in anxious patients. We found only four studies which measured biomarkers in anxiety disorder patients with regards to CHD outcome. Of the four, only two were specifically designed to address this question. Plasma lipid levels, CRP, cortisol, norepinephrine, body mass index, blood pressure and heart rate variability were mainly similar between anxious patients and controls. No study has measured thyroid hormones and BDNF. Although based on very limited data, coronary flow reserve and coronary artery calcium seem to be plausible biomarkers underlying high rate of CHD in anxiety disorder patients. The door is wide open for future studies on the subject so that hints into novel treatment options may be explored for patients suffering from both anxiety and CHD. Future studies should focus on wider range of biomarkers with sufficient statistical power. It is also an urgency to include equivalent number of women in these studies, since heart disease is the number one killer in women [42] and that there is a known gender difference in the clinical presentation (Appendix) [43].

\section{References}

1. Kessler RC, Berglund P, Demler O, Jin R, Merikangas KR (2005) Lifetime prevalence and age-of-onset distributions of DSM-IV disorders in the National Comorbidity Survey Replication. Arch Gen Psychiatry. 62(6): 593-602.

2. Chen YY, Xu P, Wang Y, Song TJ, Luo N (2019) Prevalence of and risk factors for anxiety after coronary heart disease: Systematic review and meta-analysis. Medicine (Baltimore) 98(38): e16973.

3. Edmondson D, Kronish IM, Shaffer JA, Falzon L, Burg MM (2013) Posttraumatic stress disorder and risk for coronary heart disease: a meta-analytic review. Am Heart J 166(5): 806-814.

4. Frasure Smith N, Lesperance F (2008) Depression and anxiety as predictors of 2-year cardiac events in patients with stable coronary artery disease. Arch Gen Psychiatry 65(1): 62-71.

5. Roger VL, Go AS, Lloyd Jones DM, Adams RJ, Berry JD, et al. (2011) Heart disease and stroke statistics--2011 update: a report from the American Heart Association. Circulation 123(4): e18-e209.

6. (2004) (WHO) WHO. Disease and injury country estimates: BURDEN OF DISEASE.

7. Riba MB, Wulsin LR, Rubenfire M (2011) Psychiatry and Heart Disease: The Mind, Brain, and Heart. In Riba MB, Wulsin LR, Rubenfire M (Eds.), Wiley-Blackwell, New Jersey, United States Pp: 272.

8. Zafar MU, Paz Yepes M, Shimbo D, Vilahur G, Burg MM, et al. (2010) Anxiety is a better predictor of platelet reactivity in coronary artery disease patients than depression. Eur Heart J 31(13): 1573-1782.

9. Rozanski A, Blumenthal JA, Davidson KW, Saab PG, Kubzansky L (2005) The epidemiology, pathophysiology, and management of psychosocial risk factors in cardiac practice: the emerging field of behavioral cardiology. J Am Coll Cardiol 45(5): 637-651.

10. Rozanski A, Blumenthal JA, Kaplan J (1999) Impact of psychological factors on the pathogenesis of cardiovascular disease and implications for therapy. Circulation 99(16): 2192-2217.

11. Smolderen KG, Buchanan DM, Gosch K, Whooley M, Chan PS, et al. (2017) Depression Treatment and 1-Year Mortality After Acute Myocardial Infarction: Insights from the TRIUMPH Registry (Translational Research Investigating Underlying Disparities in Acute Myocardial Infarction Patients' Health Status). Circulation 135(18): 1681-1689.

12. Kim JM, Stewart R, Lee YS, Lee HJ, Kim MC, et al. (2018) Effect of Escitalopram vs Placebo Treatment for Depression on Long-term Cardiac Outcomes in Patients with Acute Coronary Syndrome: A Randomized Clinical Trial. Jama 320(4): 350-358.

13. Copeland WE, Shanahan L, Worthman C, Angold A, Costello EJ (2012) Generalized anxiety and C-reactive protein levels: a prospective, longitudinal analysis. Psychol Med 42(12): 2641-2650.

14. Naude PJW, Roest AM, Stein DJ, de Jonge P, Doornbos B (2018) Anxiety disorders and CRP in a population cohort study with 54,326 participants: The LifeLines study. World J Biol Psychiatry 19(6): 461-470.

15. Bathla M, Singh M, Relan P (2016) Prevalence of anxiety and depressive symptoms among patients with hypothyroidism. Indian J Endocrinol Metab 20(4): 468-474.

16. Udovcic M, Pena RH, Patham B, Tabatabai L, Kansara A (2017) Hypothyroidism and the Heart. Methodist Debakey Cardiovasc J 13(2): 55-59.

17. Huo Y, Chu Y, Guo L, Liu L, Xia X (2017) Cortisol is associated with low frequency of interleukin 10-producing $B$ cells in patients with atherosclerosis. Cell Biochem Funct 35(3): 178-183.

18. Suliman S, Hemmings SM, Seedat S (2013) Brain-Derived Neurotrophic Factor (BDNF) protein levels in anxiety disorders: systematic review and meta-regression analysis. Front Integr Neurosci 7: 55. 
19. Wan R, Weigand LA, Bateman R, Griffioen K, Mendelowitz D (2014) Evidence that BDNF regulates heart rate by a mechanism involving increased brainstem parasympathetic neuron excitability. J Neurochem 129(4): 573-580.

20. Fioranelli M, Bottaccioli AG, Bottaccioli F, Bianchi M, Rovesti M (2018) Stress and Inflammation in Coronary Artery Disease: A Review Psychoneuroendocrineimmunology-Based. Front Immunol 9: 2031.

21. Martens EJ, de Jonge P, Na B, Cohen BE, Lett H (2010) Scared to death? Generalized anxiety disorder and cardiovascular events in patients with stable coronary heart disease: The Heart and Soul Study. Arch Gen Psychiatry 67(7): 750-758.

22. Ahmadi N, Hajsadeghi F, Mirshkarlo HB, Budoff M, Yehuda R (2011) Post-traumatic stress disorder, coronary atherosclerosis, and mortality. Am J Cardiol 108(1): 29-33.

23. Vaccarino V, Goldberg J, Rooks C, Shah AJ, Veledar E, et al. (2013) Posttraumatic stress disorder and incidence of coronary heart disease: a twin study. J Am Coll Cardiol 62(11): 970-978.

24. Moher D, Liberati A, Tetzlaff J, Altman DG, Group P (2009) Preferred reporting items for systematic reviews and meta-analyses: the PRISMA statement. PLoS Med 6(7): e1000097.

25. Wells G, Shea B, O Connell D, Peterson j, Welch V, et al. (2000) The Newcastle-Ottawa Scale (NOS) for Assessing the Quality of NonRandomized Studies in Meta-Analysis.

26. Zhao Q Li J, Yang J, Li R (2017) Association of total cholesterol and HDL-C levels and outcome in coronary heart disease patients with heart failure. Medicine (Baltimore) 96(9): e6094.

27. Peng J, Luo F, Ruan G, Peng R, Li X (2017) Hypertriglyceridemia and atherosclerosis. Lipids Health Dis 16(1): 233.

28. Clark LT (1986) Cholesterol and heart disease: current concepts in pathogenesis and treatment. J Natl Med Assoc 78(8): 743-751.

29. Pepys MB, Baltz ML (1983) Acute phase proteins with special reference to C-reactive protein and related proteins (pentaxins) and serum amyloid A protein. Adv Immunol 34: 141-212.

30. Ridker PM (2005) C-reactive protein, inflammation, and cardiovascular disease: clinical update. Tex Heart Inst J 32(3): 384-386.

31. Neves PO, Andrade J, Moncao H (2017) Coronary artery calcium score: current status. Radiol Bras. 50(3):182-189.

32. Chen Y, Copeland WK, Vedanthan R, Grant E, Lee JE, et al. (2013) Association between body mass index and cardiovascular disease mortality in east Asians and south Asians: pooled analysis of prospective data from the Asia Cohort Consortium. BMJ 347: f5446.

33. Khan SS, Ning H, Wilkins JT, Allen N, Carnethon M, et al. (2018) Association of Body Mass Index with Lifetime Risk of Cardiovascular Disease and Compression of Morbidity. JAMA Cardiol 3(4): 280-287.

34. Kokubo Y (2014) Prevention of hypertension and cardiovascular diseases: a comparison of lifestyle factors in Westerners and East Asians. Hypertension 63(4): 655-660.

\section{ISSN: 2574-1241}

\section{DOI: 10.26717/BJSTR.2019.23.003844}

Judith Brouillette. Biomed J Sci \& Tech Res

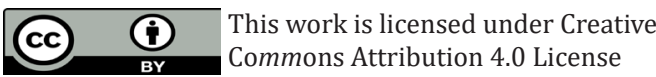

Submission Link: https://biomedres.us/submit-manuscript.php
35. Messerli FH, Fischer U, Rimoldi SF, Bangalore S (2017) Hypertension control and cardiovascular disease. Lancet 389(10065): 153.

36. Kjeldsen SE (2018) Hypertension and cardiovascular risk: General aspects. Pharmacol Res 129: 95-99.

37. Carney RM, Blumenthal JA, Stein PK, Watkins L, Catellier D, et al. (2001) Depression, heart rate variability, and acute myocardial infarction. Circulation 104(17): 2024-2028.

38. Grossman F, Potter WZ (1999) Catecholamines in depression: a cumulative study of urinary norepinephrine and its major metabolites in unipolar and bipolar depressed patients versus healthy volunteers at the NIMH. Psychiatry Res 87(1): 21-27.

39. Otte C, Neylan TC, Pipkin SS, Browner WS, Whooley MA (2005) Depressive symptoms and 24-hour urinary norepinephrine excretion levels in patients with coronary disease: findings from the Heart and Soul Study. Am J Psychiatry 162(11): 2139-2145.

40. Otte C, Marmar CR, Pipkin SS, Moos R, Browner WS (2004) Depression and 24-hour urinary cortisol in medical outpatients with coronary heart disease: The Heart and Soul Study. Biol Psychiatry 56(4): 241-247.

41. Remes O, Brayne C, van der Linde R, Lafortune L (2016) A systematic review of reviews on the prevalence of anxiety disorders in adult populations. Brain Behav 6(7): e00497.

42. Mosca L, Hammond G, Mochari Greenberger H, Towfighi A, Albert MA, et al. (2013) Fifteen-year trends in awareness of heart disease in women: results of a 2012 American Heart Association national survey. Circulation127(11): 1254-1263.

43. Collins P, Vitale C, Spoletini I, Barbaro G (2011) Gender differences in the clinical presentation of heart disease. Curr Pharm Des17(11): 10561058.

44. Dzubur Kulenovic A, Kucukalic A, Malec D (2008) Changes in plasma lipid concentrations and risk of coronary artery disease in army veterans suffering from chronic posttraumatic stress disorder. Croat Med J. 49(4): 506-514.

45. Solter V, Thaller V, Karlovic D, Crnkovic D (2002) Elevated serum lipids in veterans with combat-related chronic posttraumatic stress disorder Croat Med J 43(6): 685-689.

46. Turner JH, Neylan TC, Schiller NB, Li Y, Cohen BE (2013) Objective evidence of myocardial ischemia in patients with posttraumatic stress disorder. Biol Psychiatry 74(11): 861-866.

47. Shen BJ, Avivi YE, Todaro JF, Spiro A, Ward KD, et al. (2008) Anxiety characteristics independently and prospectively predict myocardial infarction in men the unique contribution of anxiety among psychologic factors. J Am Coll Cardiol 51(2): 113-119.

48. Park C, Guallar E, Linton JA, Lee DC, Jang Y, et al. (2013) Fasting glucose level and the risk of incident atherosclerotic cardiovascular diseases. Diabetes Care 36(7): 1988-1993.

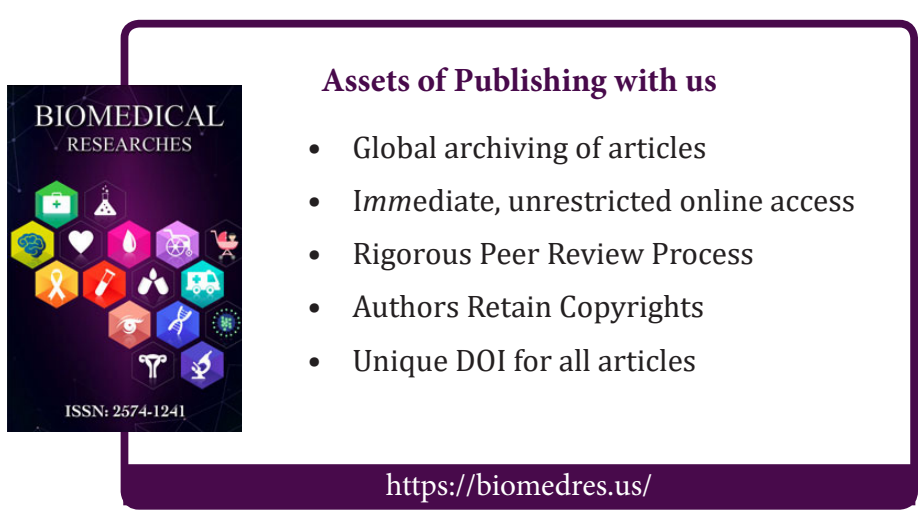

